\title{
A Note on a Model for the Quasilinear Wave Equation
}

\author{
J. Greenberg \& G. Hedstrom \\ Communicated by $\mathrm{C}$. TRUESDELL
}

\section{Introduction}

Many investigators have attempted to establish the connection between solutions of the quasilinear wave equation

$$
\frac{\partial^{2} \chi}{\partial t^{2}}=\frac{\partial}{\partial x} \sigma\left(\frac{\partial \chi}{\partial x}\right), \quad-\infty<x<\infty, t>0
$$

and the discrete system

$$
h \ddot{\chi}_{k}^{h}=\sigma\left(\frac{\chi_{k+1}^{h}-\chi_{k}^{h}}{h}\right)-\sigma\left(\frac{\chi_{k}^{h}-\chi_{k}^{h}-1}{h}\right), \quad k=0, \pm 1, \ldots, t>0 . \text { * }
$$

One problem is whether the function

$$
\chi(x, t) \equiv \lim _{\substack{h \rightarrow 0^{+} \\ k h=x}} \chi_{k}^{h}(t)
$$

is well defined and satisfies $(\mathrm{E})$ in some generalized sense. Another is whether the limits

exist and satisfy

$$
\lim _{\substack{h \rightarrow 0^{+} \\ k h=x}}\left(\dot{\chi}_{k}^{h}, \frac{\chi_{k+1}^{h}-\chi_{k}^{h}}{h}\right)
$$

$$
\lim _{\substack{h \rightarrow 0^{+} \\ h h=x}}\left(\dot{\chi}_{k}^{h}, \frac{\chi_{k+1}^{h}-\chi_{k}^{h}}{h}\right)=\left(\chi_{t}, \chi_{x}\right)
$$

at those points $(x, t)$ where $\chi$ is differentiable.

We shall answer these questions for an equation which models (E). To motivate the choice of the model equation we are forced to say a few words about the nature of the unknown $\chi$.

The function $\chi(x, t)$ represents the position at time $t$ of a material point which was located at $x$ at $t=0$. Similarily, $\chi_{k}^{h}(t)$ gives the position at time $t$ of a particle (of mass $h$ ) which was located at $k h$ at $t=0$. We envision these particles as moving on a line and therefore insist that the following inequalities be satisfied for all

\footnotetext{
* $C f$. Von Neumann [1], Ludford, Polachex, \& Seeger [2], Geiringer [3].
} 
$t \geqq 0$ :

and

$$
\chi\left(x_{2}, t\right)-\chi\left(x_{1}, t\right) \geqq 0, \quad x_{2} \geqq x_{1},
$$

$$
\chi_{k+1}^{h}(t) \geqq \chi_{k}^{h}(t), \quad k=0, \pm 1, \ldots .
$$

(1) and $(1)_{h}$ are merely the statement that mass points or particles may not pass through one another.

To obtain the discrete model equation, we replace $(E)_{h}$ by

whenever $t$ is such that

$$
\ddot{\chi}_{k}^{h}(t)=0
$$

$$
\chi_{k-1}^{h}(t)<\chi_{k}^{h}(t)<\chi_{k+1}^{h}(t) .
$$

That $(\mathrm{M})_{h}$ is true whenever $(2)_{h}$ holds amounts to the assumption that intermollecular forces are zero unless particles are in contact.

We shall supplement $(\mathrm{M})_{h}$ with the assumption that collisions are elastic.

Recall, particle $k$ is said to collide with particle $k+1$ at time $t$ if $\chi_{k}^{h}(t)=\chi_{k+1}^{h}(t)$. If we let $v_{k}^{-}\left(v_{k+1}^{-}\right)$be the velocity of the $k^{\text {th }}$ particle $\left(k+1^{\text {st }}\right.$ particle) before the collision and $v_{k}^{+}\left(v_{k+1}^{+}\right)$the velocity of the $k^{\text {th }}$ particle $\left(k+1^{\text {st }}\right.$ particle $)$ after the collision, then the assumption that collisions are elastic implies

$$
v_{k}^{+}=v_{k+1}^{-} \text {and } v_{k+1}^{+}=v_{k}^{-} \text {. }
$$

Our main result deals with the limiting behavior of solutions of the initial value problem: $(\mathrm{M})_{h}$, elastic collisions, and the initial conditions:

$$
\chi_{k}^{h}(0)=k h, \quad k=0, \pm 1, \ldots, \quad \text { and } \quad \dot{\chi}_{k}^{h}(0)= \begin{cases}a, & k=0,-1,-2, \ldots \\ b, & k=1,2, \ldots\end{cases}
$$

where $a>b$. We obtain the following results:

1) The function $\chi(x, t) \equiv \lim _{\substack{h \rightarrow 0^{+} \\ k h=x}} \chi_{k}^{h}(t)$ is well defined and Lipschitz continous

for all $x$ and all $t \geqq 0$. $\chi$ is differentiable in $x$ and $t$ except across the lines $x= \pm(a-b) t, t \geqq 0$, satisfies

$$
\chi_{t t}(x, t)=0 \text { when } \quad x \neq \pm(a-b) t, \quad t>0,
$$

and satisfies the initial conditions:

$$
\chi(x, 0)=x \text { and } \chi_{t}(x, 0)=\left\{\begin{array}{ll}
a, & x<0 \\
b, & x>0
\end{array}\right\} .
$$

2) For points $(x, t)$ satisfying $|x|>(a-b) t, t>0$, the limits

$$
\lim _{\substack{h \rightarrow 0^{+} \\ k h=x}} \dot{\chi}_{k}^{h}(t) \text { and } \lim _{\substack{h \rightarrow 0^{+} \\ k h=x}} \frac{\chi_{k+1}^{h}(t)-\chi_{k}^{h}(t)}{h}
$$

exist and equal $\chi_{t}(x, t)$ and $\chi_{x}(x, t)$ respectively. For points $(x, t)$ such that $|x|<(a-b) t, t>0$ the above limits fail to exist. 
3) The discrete motions $\chi_{k}^{h}(t)$ are decomposable by averaging into a slowly varying component $\Psi_{k}^{h}(t)$ and a rapidly varying component $h \eta_{k}^{h}(t)$; i.e.

where

$$
\chi_{k}^{h}(t)=\Psi_{k}^{h}(t)+h \eta_{k}^{h}(t)
$$

$$
\dot{\Psi}_{k}^{h}(t)=O(1), \quad \eta_{k}^{h}(t)=O(1), \quad \text { and } \quad \dot{\eta}_{k}^{h}(t)=O(1 / h) .
$$

Moreover, $\Psi_{k}^{h}$ has the additional property that

and

$$
\lim _{\substack{h \rightarrow 0^{+} \\ k h=x}} \Psi_{k}^{h}(t)=\chi(x, t),-\infty<x<\infty, t \geqq 0 ;
$$

$$
\lim _{\substack{h \rightarrow 0^{+} \\ k h=x}} \dot{\Psi}_{k}^{h}(t)=\chi_{t}(x, t) \text { and } \lim _{\substack{h \rightarrow 0^{+} \\ k h=x}} \frac{\Psi_{k+1}-\Psi_{k}}{h}=\chi_{x}
$$

for all $x$ and $t>0$ such that $x \neq \pm(a-b) t$.

It should be observed that the lines $x= \pm(a-b) t, t \geqq 0$ play the role of shock waves for the function $\chi$.

\section{Proofs}

For each $h>0$ and each $a>b$ we let $\Delta=\frac{h}{a-b}$. We define the continuous function

$$
J_{0}^{h}(t) \equiv\left\{\begin{array}{l}
n(a+b) \Delta+a(t-2 n \Delta), 2 n \Delta \leqq t \leqq(2 n+1) \Delta, \\
(n+1) a \Delta+n b \Delta+b(t-(2 n+1) \Delta),(2 n+1) \Delta \leqq t \leqq 2(n+1) \Delta, \\
n=0,1,2, \ldots
\end{array}\right.
$$

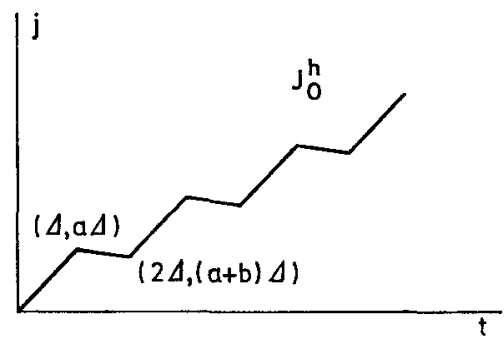

Fig. 1

We shall now describe the solution of the initial value problem stated in Section 1.

For $k \leqq 0$, the particle trajectories $\chi_{k}^{h}(t)$ are given by

and for $k \geqq 1$ by

$$
\chi_{k}^{h}(t)= \begin{cases}k h+a t, & 0 \leqq t \leqq-k \Delta, \\ k h-a k \Delta+J_{0}^{h}(t+k \Delta), & t \geqq-k \Delta,\end{cases}
$$

$$
\chi_{k}^{h}(t)= \begin{cases}k h+b t, & 0 \leqq t \leqq k \Delta, \\ k h+b k \Delta+J_{h}^{0}(t-k \Delta), & t \geqq k \Delta .\end{cases}
$$


It follows from the last set of formulas that

exists and is given by

$$
\chi(x, t)=\lim _{\substack{k \rightarrow 0 \\ k h=x}} \chi_{k}^{h}(t)
$$

$$
\chi(x, t)=\left\{\begin{array}{lll}
x+a t, & x \leqq-(a-b) t, & t \geqq 0, \\
\frac{a+b}{2} t+\frac{x}{2}, & -(a-b) t \leqq x \leqq(a-b) t, & t \geqq 0, \\
x+b t, & (a-b) t \leqq x, & t \geqq 0 .
\end{array}\right.
$$

That $\chi$ satisfies $\chi_{t t}=0$ when $x \neq \pm(a-b) t$, and the initial conditions

is immediate.

$$
\chi(x, 0)=x \text { and } \chi_{i}(x, 0)=\left\{\begin{array}{ll}
a, & x<0 \\
b, & x>0
\end{array}\right\}
$$

That the limits

$$
\lim _{\substack{h \rightarrow 0^{+} \\ k h=x}} \dot{\chi}_{k}^{h}(t) \text { and } \lim _{\substack{h \rightarrow 0^{+} \\ k h=x}} \frac{\chi_{k+1}^{h}-\chi_{k}^{h}}{h}
$$

have the proper value for $|k h|=|x|>(a-b) t$ is a direct consequence of equations (2.2) and (2.3).

That the limits of these same quantities fail to exist when $|k h|=|x|<(a-b) t$ follows from (2.2) and (2.3) and the oscillitatory character of $J_{0}^{h}$ (see (2.1)).

The existence of the slowly varying and rapidly varying decomposition of the trajectories $\chi_{k}^{h}(t)$ is a consequence of the observation that

and therefore that

$$
\frac{d J_{0}^{h}}{d t}(t+2 d)=\frac{d J_{0}^{h}}{d t}(t), \quad t \geqq 0,
$$

$$
\frac{d \chi_{k}^{h}}{d t}(t+2 \Delta)=\frac{d \chi_{k}^{h}}{d t}(t), \quad t \geqq|k| \Delta
$$

We define the slowly varying flow

$$
\Psi_{k}^{h}(t)=k h-\frac{1}{2 \Delta} \int_{0}^{2 \Delta} \chi_{k}^{h}(s) d s+\frac{1}{2 \Delta} \int_{t}^{t+2 \Delta} \chi_{k}^{h}(s) .
$$

A routine computation shows that the difference between $\chi_{k}^{h}(t)$ and $\Psi_{k}^{h}(t)$ may be written as $h \eta_{k}^{h}(t)$ where $\eta_{k}^{h}$ is $O(1)$ and has a time derivative $\dot{\eta}_{k}^{h}=O(1 / h)$.

We shall now show that

$$
\lim _{\substack{k \rightarrow 0^{+} \\ k h=x}} \dot{\Psi}_{k}^{h}(t)=\frac{a+b}{2}=\chi_{t}(x, t)
$$

whenever $|x|<(a-b) t$. The result for the spacial derivatives follows from similar arguments. We have

$$
\frac{d \Psi_{k}^{h}}{d t}=\frac{\chi_{k}^{h}(t+2 \Delta)-\chi_{k}^{h}(t)}{2 \Delta}=\frac{1}{2 \Delta} \int_{t}^{t+2 \Delta} \dot{\chi}_{k}^{h}(s) d s
$$


But, for $|x|=|k h|<(a-b) t$ we know that $\dot{\chi}_{k}^{h}(t)$ is $2 \Delta$ periodic. In fact we know that over any interval $(t, t+2 \Delta)$ with $t>\frac{|k h|}{(a-b)} \dot{\chi}_{k}^{h}$ is equal to a over half of the interval and is equal to $b$ over the other half. Hence, for $t>\frac{|k h|}{(a-b)}$

$$
\frac{d \Psi_{k}^{h}}{d t}=\frac{a+b}{2} \text {. Q.E.D. }
$$

\section{Concluding Remarks}

We shall now make a number of observations which contrast the behavior of solutions of the limiting continuous system to the behavior of solutions of the particulate system from which it was derived.

Our first observation is that the limiting solution is dissipative while each comparable particulate system is conservative. We shall demonstrate this by the following elementary computation. We let $L>0$ be any positive number, and we let

$$
E(L, t)=\frac{1}{2} \int_{-L}^{L} \dot{\chi}_{t}^{2}(x, t) d x .
$$

The quantity $E(L, t)$ is merely the kinetic energy at time $t$ of those mass points located in the interval $(-L, L)$ at $t=0$. A simple computation shows that

$$
\begin{aligned}
E(L, t) & =\frac{L}{2}\left(a^{2}+b^{2}\right)-\frac{t}{4}(a-b)^{3}, & & 0 \leqq t \leqq \frac{L}{a-b} \\
& =\frac{L}{4}(a+b)^{2}, & & \frac{L}{a-b} \leqq t<\infty .
\end{aligned}
$$

Now, let $h$ be any positive number. $\chi_{k}^{h}(t)$ will be the solution of the particulate problem (see equations (2.2) and (2.3)) and

$$
E^{h}(L, t) \equiv \frac{1}{2} \sum_{k=-\left[\frac{L}{h}\right]-1}^{\left[\frac{L}{h}\right]+1} h\left(\dot{\chi}_{k}^{h}(t)\right)^{2}
$$

is the kinetic energy at time $t$ of all particles (each of mass $h$ ) located in the interval $\left[-\left[\frac{L}{h}\right]-1,\left[\frac{L}{h}\right]+1\right]$ at $t=0$. A quick computation shows that

$$
\left|E^{h}(L, t)-\frac{L}{2}\left(a^{2}+b^{2}\right)\right| \leqq\left(a^{2}+b^{2}\right) h
$$

for all times $t$ and hence that

$$
E^{0}(L, t) \equiv \lim _{h \rightarrow 0^{+}} E^{h}(L, t)=\frac{L}{2}\left(a^{2}+b^{2}\right), \quad t \geqq 0 .
$$

A comparison of (3.2) to (3.5) yields one example of the dissipative nature of the limiting continuous system. 
Similar computations show that for any $x_{1}<x_{2}$ and any time $t$

$$
E\left(x_{1}, x_{2}, t\right) \equiv \frac{1}{2} \int_{x_{1}}^{x_{2}} \chi_{t}^{2}(x, t) d x \leqq E^{0}\left(x_{1}, x_{2}, t\right) \equiv \lim _{h \rightarrow 0^{+}} \frac{1}{2} \sum_{k=\left[\frac{x_{1}}{h}\right]-1}^{\left[\frac{x_{2}}{h}\right]+1} h\left(\dot{\chi}_{k}^{h}(t)\right)^{2}
$$

with strict inequality holding if $t>\min \left(\frac{\left|x_{1}\right|,\left|x_{2}\right|}{a-b}\right)$.

Finally, we note that the limit flow $\chi$ defined in (2.4) is a non-standard solution of the Burger-Hopf Equation

$$
v_{t}+v v_{y}=0 \text {. }
$$

That is if we let $v(y, t) \equiv \chi_{t}(x, t)$ when $y=\chi(x, t)$, then $v$ satisfies (BH) almost everywhere together with the initial conditions

$$
v(y, 0)=\left\{\begin{array}{ll}
a, & y<0 \\
b, & y>0
\end{array}\right\}
$$

The normally accepted solutions to this problem is

Our solution is

$$
v(y, t)=\left\{\begin{array}{ll}
a, & y<\frac{a+b}{2} t \\
b, & y>\frac{a+b}{2} t
\end{array}\right\} .
$$

$$
v(y, t)=\left\{\begin{array}{lc}
a, & y<b t, \\
\frac{a+b}{2}, & b t<y<a t, \\
b, & a t<y .
\end{array}\right.
$$

We point out that the normally accepted solution to this problem would have been obtained as a limiting solution of our discrete system had we imposed the condition that all collisions were plastic rather than elastic.

\section{References}

1. Von Neumann, J., Proposal and Analysis of a New Numerical Method for the Treatment of Hydrodynamical Shock Problems. Reprinted in the collected works of John von Neumann, Vol. 6. New York: Macmillan Company 1963.

2. Ludford, G., H. PolACHEK, \& R. J. SEeger, On unsteady flows of compressible viscous fluids. J. Appl. Phys. 24, 490-495 (1953).

3. GeIrInger, H., On Numerical Methods in Wave Interaction Problems. Advances in Applied Mechanics, Vol. 1. New York: Academic Press 1948.

Department of Mathematics University of Michigan and

Department of Mathematics Case-Western Reserve University 